IJMMS 31:1 (2002) 31-36

PII. S016117120201284X

http://ijmms.hindawi.com

(c) Hindawi Publishing Corp.

\title{
SEQUENCES AND SERIES INVOLVING THE SEQUENCE OF COMPOSITE NUMBERS
}

\author{
PANAYIOTIS VLAMOS
}

Received 17 April 2001 and in revised form 2 October 2001

Denoting by $p_{n}$ and $c_{n}$ the $n$th prime number and the $n$th composite number, respectively, we prove that both the sequence $\left(x_{n}\right)_{n \geq 1}$, defined by $x_{n}=\sum_{k=1}^{n}\left(c_{k+1}-c_{k}\right) / k-p_{n} / n$, and the series $\sum_{n=1}^{\infty}\left(p_{c_{n}}-c_{p_{n}}\right) / n p_{n}$ are convergent.

2000 Mathematics Subject Classification: 11A25.

1. Introduction. We use the following notation:

(i) $\pi(x)$ is the number of prime numbers less than or equal to $x$,

(ii) $p_{n}$ is the $n$th prime number,

(iii) $c_{n}$ is the $n$th composite number; $c_{1}=4, c_{2}=6, \ldots$,

(iv) $\log _{2} n=\log (\log n)$.

In 1967, Bojarincev [1] estimated $c_{n}$ and found out that

$$
c_{n}=n\left(1+\frac{1}{\log n}+\frac{2}{\log ^{2} n}+\frac{4}{\log ^{3} n}+\frac{19}{2} \frac{1}{\log ^{4} n}+\frac{181}{6} \frac{1}{\log ^{5} n}+o\left(\frac{1}{\log ^{5} n}\right)\right) .
$$

For $c_{n}^{(1)}:=c_{n}$ and $c_{n}^{(k+1)}:=c_{c_{n}^{(k)}}, k \geq 1$, we can prove that

$$
c_{n}^{(k+1)}-2 c_{n}^{(k)}+c_{n}^{(k-1)} \sim \frac{n}{\log ^{2} n} .
$$

If $n$ is large enough, then

$$
c_{n}^{(k)}>\sqrt{c_{n}^{(k-1)} \cdot c_{n}^{(k+1)}} .
$$

It was shown in [3] that, for $n$ large enough we have

$$
p_{c_{n}}>c_{p_{n}}
$$

Of course, the irregularities in the distribution of the prime numbers imply irregularities in the distribution of the composed numbers. Although in the sequence of the composite numbers, large "gaps" cannot be found. For the sequence $\left(p_{n}\right)_{n \geq 1}$ we have $\limsup _{n \rightarrow \infty}\left(p_{n+1}-p_{n}\right)=\infty$, while in the case of the sequence $\left(c_{n}\right)_{n \geq 1}$ we have $1 \leq c_{k+1}-c_{k} \leq 2$, with the specification that $c_{k+1}-c_{k}=2$ if and only if the number $c_{k+1}$ is prime. In this case, denoting $c_{k}+1=p_{m}$, it is proved in [3] that

$$
k=k(m)=p_{m}+m x_{m} \quad \text { with } \lim _{m \rightarrow \infty} x_{m}=1 .
$$


Since $c_{n} \sim n$, we can expect that "in mean" the sequence $\left(c_{n}\right)_{n \geq 1}$ behaves as the sequence of the natural numbers. It is readily seen that, since the series $\sum_{n=1}^{\infty} 1$ / $n\left(p_{n}-p_{n+1}\right)$ is divergent, it follows that the series $\sum_{n=1}^{\infty} 1 / c_{n}\left(p_{n}-p_{n+1}\right)$ is divergent too.

The situations to be analyzed in the present paper are, however, more complicated and lean on a series of facts, namely

$$
\begin{gathered}
\lim _{n \rightarrow \infty}\left(\sum_{k=1}^{n} \frac{1}{k}-\log n\right)=\gamma ; \\
\text { the series } \sum_{n=2}^{\infty} \frac{1}{n(\log n)^{\alpha}} \text { is convergent, for } \alpha>1 \\
\pi(x)=\int_{2}^{x} \frac{d t}{\log t}+O\left(\frac{x}{\log ^{k} x}\right), \quad \text { for every } k>0 \\
p_{n}=n\left(\log n+\log _{2} n-1+\frac{\log _{2} n-2}{\log n}+O\left(\frac{\log _{2}^{2} n}{\log ^{2} n}\right)\right) \quad \text { (see [2]); } \\
\log p_{n}=\log n+\log _{2} n+\frac{\log _{2} n-1}{\log n}+O\left(\frac{\log _{2}^{2} n}{\log ^{2} n}\right) \quad \text { (see [5]); } \\
b=\lim _{n \rightarrow \infty}\left(\sum_{k=2}^{n} \frac{1}{p_{k}}-\log \log n\right) \quad\left(\operatorname{see}^{2}[4]\right)
\end{gathered}
$$

\section{Asymptotic behavior of certain series}

Proposition 2.1. The sequence $x_{n}=\sum_{k=1}^{n}\left(c_{k+1}-c_{k}\right) / k-p_{n} / n$ is convergent.

Proof. We have

$$
x_{n}=\sum_{k=1}^{n} \frac{1}{k}+\sum_{k \leq n}^{\prime} \frac{1}{k}-\frac{p_{n}}{n},
$$

where $\sum^{\prime}$ extends to all values of $k$ such that $c_{k}+1$ is a prime number, that is, $c_{k}+1=$ $p_{m}, m=3,4, \ldots, \pi(n)$. It follows by $(1.5)$ that

$$
\sum_{k=1}^{n} \frac{1}{k}=\sum_{m=3}^{\pi(n)} \frac{1}{p_{m}+m x_{m}}=\sum_{m=3}^{\pi(n)} \frac{1}{p_{m}}-\sum_{m=3}^{\pi(n)} \frac{m x_{m}}{p_{m}\left(p_{m}+m x_{m}\right)} .
$$

Since $p_{m} \sim m \log m$, we get $m x_{m} / p_{m}\left(p_{m}+m x_{m}\right) \sim 1 m \log ^{2} m$ and then (1.7) implies that the series $\sum_{m=1}^{\infty} m x_{m} / p_{m}\left(p_{m}+m x_{m}\right)$ is convergent; denote its sum by $a$. In view of (1.11), it then follows that

$$
\sum_{k=1}^{n} \frac{1}{k}-\log _{2} n \stackrel{n \rightarrow \infty}{\longrightarrow} b-a
$$

By (1.10) $\lim _{n \rightarrow \infty}\left(p_{n} / n-\log n-\log _{2} n\right)=-1$, hence (1.6) and (2.3) imply that

$$
\lim _{n \rightarrow \infty} x_{n}=\gamma+b-a+1 \text {. }
$$

The proof is completed. 
REMARK 2.2. It follows by Proposition 2.1 that both the series $\sum_{k=1}^{\infty}\left(c_{k+1}-c_{k}\right) / k$ and $\sum_{k=1}^{\infty}\left(c_{k+1}-c_{k}-1\right) / k$ are divergent.

Proposition 2.3. Let $s$ be a real number. If $y_{n}=\sum_{k=1}^{n}\left(c_{k+1}-c_{k}\right)^{s} / k$, then $y_{n}=$ $\log n+\left(2^{s}-1\right) \log _{2} n+O(1)$.

Proof. If the number $c_{k}+1$ is composed, then $c_{k+1}-c_{k}=1$, while if $c_{k}+1$ is prime then $c_{k+1}-c_{k}=2$. Thus

$$
y_{n}=\sum_{k=1}^{n} \frac{1}{k}+\sum_{k=1}^{n} \frac{2^{s}-1}{k},
$$

where $\sum^{\prime}$ extends to the indices $k$ such that $c_{k}+1$ is prime. Now (1.6) and (2.3) imply that

$$
y_{n}=\log n+\gamma+o(1)+\left(2^{s}-1\right)\left(\log _{2} n+b-a+o(1)\right)
$$

and the proof ends.

Proposition 2.4. If $z_{n}=\sum_{k=1}^{n}\left(c_{k+1}-c_{k}\right) /\left(c_{k+2}-c_{k+1}\right)$, then $z_{n}=n+3 / 2 \cdot n / \log n+$ $O\left(n / \log ^{2} n\right)$.

Proof. The following cases can arise:

(a) if both $c_{k+1}$ and $c_{k+1}+1$ are composite numbers, then $c_{k+1}-c_{k}=c_{k+2}-c_{k+1}=1$;

(b) if $c_{k+1}$ is prime and $c_{k+1}+1$ is composed, then $c_{k+1}-c_{k}=2$ and $c_{k+2}-c_{k+1}=1$;

(c) if $c_{k+1}$ is composite and $c_{k+1}+1$ is prime, then $c_{k+1}-c_{k}=1$ and $c_{k+2}-c_{k+1}=2$;

(d) if both $c_{k+1}$ and $c_{k+1}+1$ are prime numbers, then $c_{k+1}-c_{k}=c_{k+2}-c_{k+1}=2$.

Next, denote by $\pi_{2}(x)$ the number of the prime numbers $p \leq x$ such that $p+2$ is prime. It is known (see [2]) that

$$
\pi_{2}(x)=O\left(\frac{x}{\log ^{2} x}\right) .
$$

By taking into consideration the above four cases, it follows that

$$
z_{n}=1(n-\pi(n))+2\left(\pi(n)-\pi_{2}(n)\right)+\frac{1}{2}\left(\pi(n)-\pi_{2}(n)\right)+\pi_{2}(n)+O(1) .
$$

Since $\pi(x)=x / \log x+O\left(x / \log ^{2} x\right)$, the desired conclusion follows.

Proposition 2.5. If $t_{n}=\sum_{k=1}^{n}\left(c_{k+2}-2 c_{k+1}+c_{k}\right)^{2}$, then $t_{n}=2 \pi(n)+O(1)$.

PROoF. By analyzing the cases occurring in the proof of the preceding proposition, we see that $c_{k+2}-2 c_{k+1}+c_{k}=0$ in the cases (a) and (d), while $\left(c_{k+2}-2 c_{k+1}+c_{k}\right)^{2}=1$ in the cases (b) and (d). Consequently, $t_{n}=2 \pi(n)+O(1)$.

\section{Studying the convergence of certain series}

Proposition 3.1. The series

$$
\sum_{n=1}^{\infty} \frac{p_{c_{n}}-c_{p_{n}}}{n p_{n}}
$$

is convergent. 
First we prove the following fact.

LEMMA 3.2. The relation

$$
\frac{1}{\log p_{n}}=\frac{1}{\log n}-\frac{\log _{2} n}{\log ^{2} n}+O\left(\frac{\log _{2}^{2} n}{\log ^{3} n}\right)
$$

holds.

Proof. By (1.10), it follows that

$$
\frac{1}{\log p_{n}}=\frac{1}{\log n} \cdot \frac{1}{1+\log _{2} n / \log n+\log _{2} n / \log ^{2} n+O\left(1 / \log ^{2} n\right)} .
$$

For $|x|<1$ we have $1 /(1+x)=1-x+O\left(x^{2}\right)$, hence,

$$
\frac{1}{\log p_{n}}=\frac{1}{\log n} \cdot\left(1-\frac{\log _{2} n}{\log n}-\frac{\log _{2} n}{\log ^{2} n}+O\left(\frac{\log _{2}^{2} n}{\log ^{2} n}\right)\right)
$$

which implies the desired conclusion.

Proof of Proposition 3.1. By (1.1), we get

$$
c_{p_{n}}=p_{n}\left(1+\frac{1}{\log p_{n}}+\frac{2}{\log ^{2} p_{n}}+O\left(\frac{1}{\log ^{3} p_{n}}\right)\right)
$$

and then we have, in view of the above lemma,

$$
c_{p_{n}}=p_{n}\left(1+\frac{1}{\log n}-\frac{\log _{2} n-2}{\log ^{2} n}+O\left(\frac{\log _{2}^{2} n}{\log ^{3} n}\right)\right) .
$$

Now, replacing $p_{n}$ by means of (1.9), we get

$$
c_{p_{n}}=p_{n}+n+\frac{n}{\log n}+O\left(\frac{n \log _{2}^{2} n}{\log ^{2} n}\right) .
$$

If we let $x=p_{c_{n}}$ in (1.8), then we deduce

$$
c_{n}=\int_{2}^{p_{c_{n}}} \frac{d t}{\log t}+O\left(\frac{n}{\log ^{2} n}\right) .
$$

For $x=c_{p_{n}}$, also (1.8) implies

$$
\pi\left(c_{p_{n}}\right)=\int_{2}^{c_{p_{n}}} \frac{d t}{\log t}+O\left(\frac{n}{\log ^{2} n}\right)
$$

It is readily seen that $\pi\left(c_{m}\right)+m+1=c_{m}$ and for $m=p_{n}$ this implies that $\pi\left(c_{p_{n}}\right)+$ $p_{n}+1=c_{p_{n}}$. Then, (3.9) becomes

$$
c_{p_{n}}-p_{n}-1=\int_{2}^{c_{p_{n}}} \frac{d t}{\log t}+O\left(\frac{n}{\log ^{2} n}\right) .
$$

By subtracting (3.8) from the above relation, we get

$$
c_{p_{n}}-p_{n}-c_{n}-1=\int_{p_{c_{n}}}^{c_{p_{n}}} \frac{d t}{\log t}+O\left(\frac{n}{\log ^{2} n}\right) .
$$


In view of (1.1) and (3.7), this relation takes the form

$$
O\left(\frac{n \log _{2}^{2} n}{\log ^{2} n}\right)=\int_{p_{c_{n}}}^{c_{p_{n}}} \frac{d t}{\log t}+O\left(\frac{n}{\log ^{2} n}\right) .
$$

By applying the mean value theorem to the above integral, it follows that there exists $\theta_{n} \in\left(p_{c_{n}}, c_{p_{n}}\right)$ such that

$$
\int_{p_{c_{n}}}^{c_{p_{n}}} \frac{d t}{\log t}=\frac{c_{p_{n}}-p_{c_{n}}}{\log \theta_{n}}
$$

Since $\log \theta_{n} \sim \log n$, (3.12) becomes

$$
O\left(\frac{n \log _{2}^{2} n}{\log ^{2} n}\right)=\frac{c_{p_{n}}-p_{c_{n}}}{\log n}+O\left(\frac{n}{\log ^{2} n}\right) .
$$

Since $p_{n} \sim n \log n$, we obtain furthermore

$$
\frac{c_{p_{n}}-p_{c_{n}}}{n p_{n}}=O\left(\frac{\log _{2}^{2} n}{n \log ^{2} n}\right)
$$

For $n$ large enough we have

$$
c_{p_{n}}-p_{c_{n}}>0, \quad \frac{\log _{2}^{2} n}{n \log ^{2} n}<\frac{1}{n \log ^{1.5} n},
$$

hence in view of (1.6), it follows that the series $\sum_{n=1}^{\infty}\left(c_{p_{n}}-p_{c_{n}}\right) / n p_{n}$ is convergent.

Proposition 3.3. Let $k \geq 3$ and let $\alpha_{1}, \alpha_{2}, \ldots, \alpha_{k}$ be real numbers such that $\sum_{i=1}^{k} \alpha_{i}=$ 0 and $\sum_{i=1}^{k}(i-1) \alpha_{i}=0$. If $\left(x_{n}\right)_{n \geq 1}$ is a decreasing sequence which converges to 0 , then the series

$$
\sum_{n=1}^{\infty}\left(\alpha_{k} c_{n+k}+\alpha_{k-1} c_{n+k-1}+\cdots+\alpha_{1} c_{n+1}\right) x_{n}
$$

is convergent.

PROoF. Denote $\varepsilon_{i}=\alpha_{k} c_{i+k}+\alpha_{k-1} c_{i+k-1}+\cdots+\alpha_{1} c_{i+1}$ and $a=\alpha_{1} c_{2}+\left(\alpha_{1}+\alpha_{2}\right) c_{3}+$ $\left(\alpha_{1}+\alpha_{2}+\cdots+\alpha_{k-1}\right) c_{k}$. Since $\sum_{i=1}^{k} \alpha_{i}=0$, it then follows that

$$
\sum_{i=1}^{k} \varepsilon_{i}=a+\alpha_{k} c_{n+k}+\left(\alpha_{k}+\alpha_{k-1}\right) c_{n+k-1}+\cdots+\left(\alpha_{k}+\alpha_{k-1}+\cdots+\alpha_{2}\right) c_{n+2} .
$$

Denote $c_{n+i}=c_{n+2}+x_{i}^{(n)}$. Since $1 \leq c_{i+1}-c_{i} \leq 2$, it then follows that $1 \leq x_{i}^{(n)}<2 k$. Relation (3.18) can take the form

$$
\begin{aligned}
\sum_{i=1}^{k} \varepsilon_{i} & =a+\sum_{i=1}^{k}(i-1) \alpha_{i} c_{n+2}+\sum_{i=3}^{k}\left(\alpha_{k}+\alpha_{k-1}+\cdots+\alpha_{i}\right) x_{i}^{(n)} \\
& =a+\sum_{i=3}^{k}\left(\alpha_{k}+\alpha_{k-1}+\cdots+\alpha_{i}\right) x_{i}^{(n)}
\end{aligned}
$$


Since

$$
\left|\sum_{i=3}^{k}\left(\alpha_{k}+\alpha_{k-1}+\cdots+\alpha_{i}\right) x_{i}^{(n)}\right| \leq \sum_{i=3}^{k}\left(\left|\alpha_{k}\right|+\left|\alpha_{k-1}\right|+\cdots+\left|\alpha_{i}\right|\right) \cdot 2 k=M,
$$

then it follows that the sequence $\left(\sum_{i=1}^{n} \varepsilon_{i}\right)_{n \geq 1}$ is bounded. Consequently, the convergence of our series follows by Dirichlet's criterion.

Proposition 3.4. The series

$$
\sum_{n=1}^{\infty} \frac{c_{n+1}-c_{n}-1}{p_{n}}
$$

is convergent.

Proof. Denoting $S_{n}=\sum_{k=1}^{n}\left(c_{k+1}-c_{k}-1\right) / p_{k}$, it follows that $S_{n}=\sum_{p_{k} \leq n}^{\prime} 1 / p_{k}$, where $\sum^{\prime}$ extends to the indices $k$ such that $c_{k}+1=p_{m}$ with prime $p_{m}$. In view of (1.5), it follows that $k=k(m) \sim p_{m} \sim m \log m$, hence $p_{k} \sim k \log k \sim m \log ^{2} m$. then (1.7) implies that the series $\sum_{n=1}^{\infty}\left(c_{n+1}-c_{n}-1\right) / p_{n}$ is convergent.

\section{REFERENCES}

[1] A. E. Bojarincev, Asymptotic expressions for the $n$th composite number, Ural. Gos. Univ. Mat. Zap. 6 (1967), 21-43.

[2] V. Brun, Le Crible d'Erathostène et le Théorème de Goldbach, vol. 3, Videnskapselkapets Skrifter I, Kristiana, 1920 (French).

[3] L. Panaitopol, Some properties of the series of composed numbers, JIPAM. J. Inequal. Pure Appl. Math. 2 (2001), no. 3, 1-6.

[4] J. B. Rosser and L. Schoenfeld, Approximate formulas for some functions of prime numbers, Illinois J. Math. 6 (1962), 64-94.

[5] P. Vlamos, Inequalities Involving the Sequence of Differences of the Prime Numbers, I, vol. 32, Publications de Centre de Recherches en Mathématiques Pures, Neuchâtel, 2001.

PANayiotis Vlamos: Hellenic Open University, PATras, Greece

E-mail address: v1amos@v1amos.com 


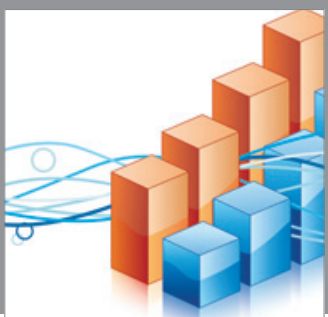

Advances in

Operations Research

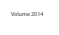

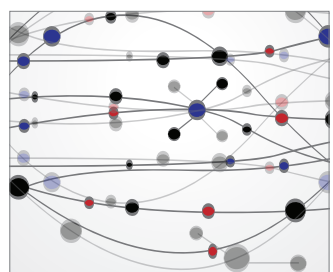

\section{The Scientific} World Journal
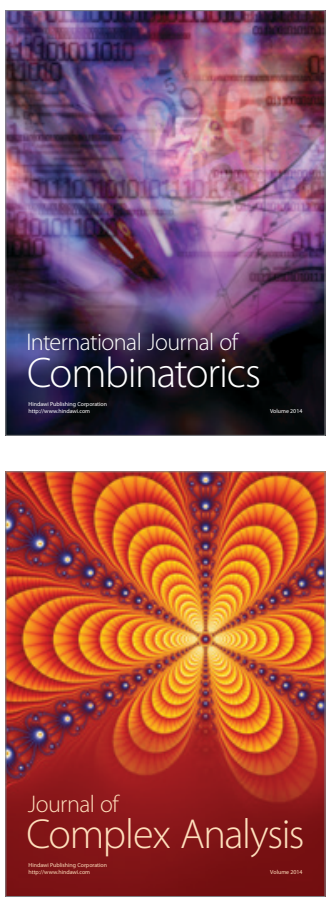

International Journal of

Mathematics and

Mathematical

Sciences
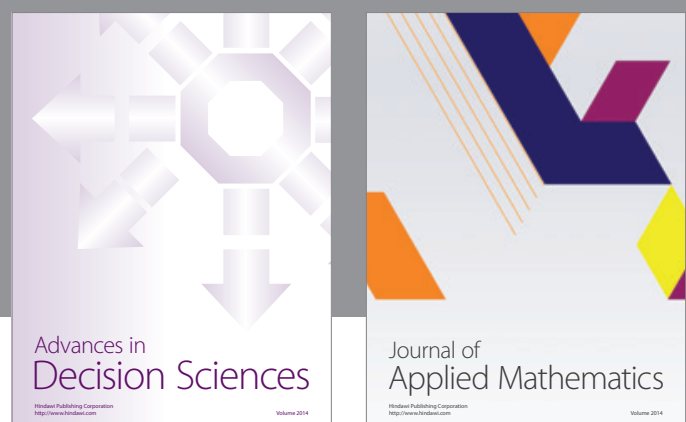

Journal of

Applied Mathematics
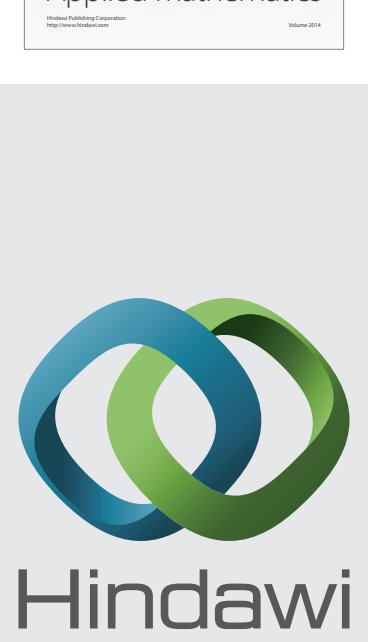

Submit your manuscripts at http://www.hindawi.com
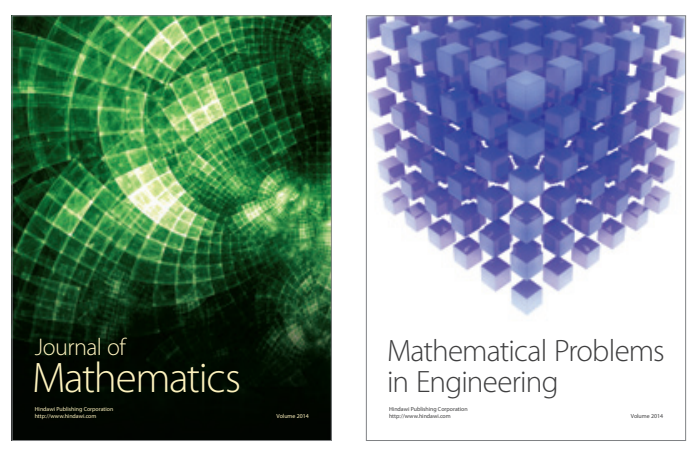

Mathematical Problems in Engineering
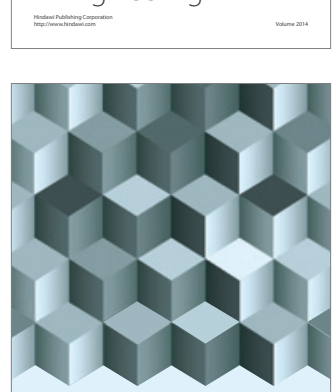

Journal of

Function Spaces
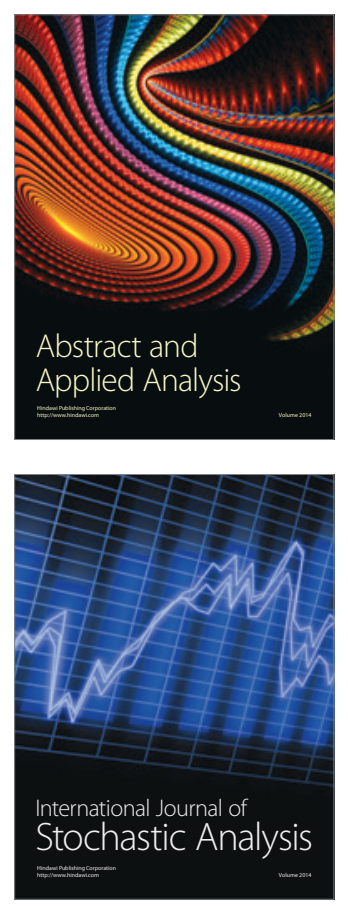

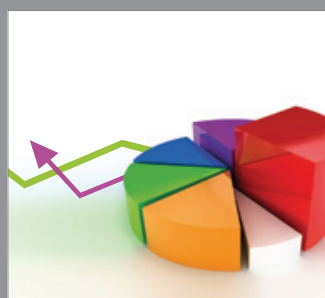

ournal of

Probability and Statistics

Promensencen
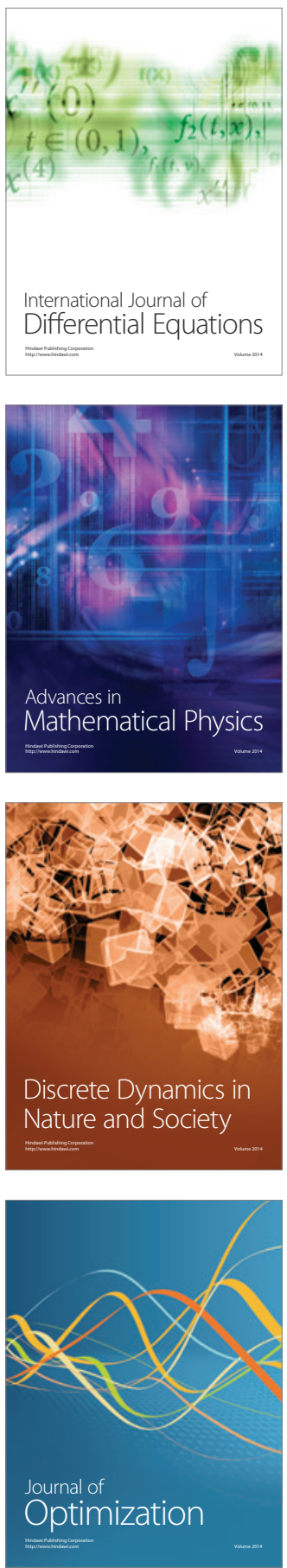